\title{
A Inteligência Artificial nos Contratos: Uma hipótese possível?
}

\section{Demétrio Beck da Silva Giannakos' Wilson Engelmann}

\section{Resumo}

O texto visa analisar se, dada a realidade brasileira, seria possível inserir nos contratos privados sistemas de Inteligência Artificial - |A. Primeiro, serão analisados os conceitos existentes sobre IA, bem como a dificuldade em conceituá-la de uma única maneira. O texto abordará, através do conceito de contrato, a possibilidade e as vantagens da utilização da IA nos contratos privados.
Tal ideia foi, primeiramente, publicada por Gustavo Tepedino e Rodrigo da Guia Silva que, então, surgiu como inspiração para a elaboração do presente trabalho, com a proposta de tentar ir além.

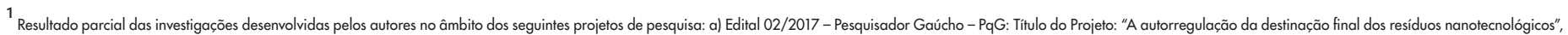

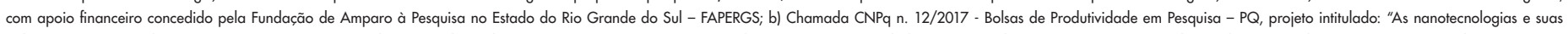

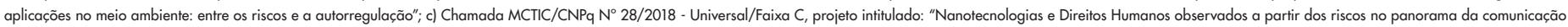

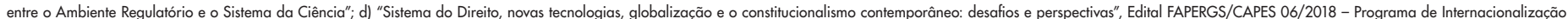

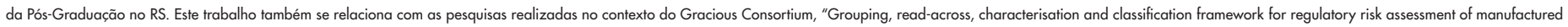
nanomaterials and safer design of nano-enabled products", com recursos financeiros do Eurpean Union's Horizon 2020 research and innovation programme under Grant Agrement n. 760840, Disponivel em: www.h2020gracious.eu
} 
Também o Tribunal observou esta dicotomia, notando que as pessoas em causa "são apresentados como o oposto dos portugueses de bem, colados a um discurso em que se fala deles e das pessoas como eles de forma depreciativa". Acresce que se tratam de "pessoas moradoras de um bairro degradado e de modesta condição social, na sua maioria vindas de países Africanos, a cuja imagem o Réu cola toda uma panóplia de menções depreciativas".

Para nós, o conflito subjacente à decisão apresentada revela-se uma oportunidade didática, na medida em que se configura como um exemplo da forma como o populismo faz uso das emoções públicas para levar a cabo os seus objetivos políticos, nomeadamente, no âmbito da política criminal. É sabido que André Ventura e o Chega não se têm imiscuído de partilhar as suas ideias para propostas legislativas de natureza penal: a pena de prisão perpétua, a pena de castração física ou química para agentes de crimes de abuso sexual de menores, o aumento em dobro do limite máximo da moldura dos crimes de corrupção - entre outras. A força do discurso populista é alimentada pela dicotomia concretizada nos termos anteriormente descritos, mas que podem bem ser alternativamente concebida como uma cisão entre os "bons" e os "maus". Assim, de forma pouco

\section{Abstract}

The text aims to analyze whether, given the Brazilian reality, it would be possible to insert Artificial Intelligence - Al systems in private contracts. First, the existing concepts about Al will be analyzed, as well as the difficulty in conceptualizing it in a single way. The text will address, through the concept of contract, the possibility and the advantages of the use of $\mathrm{Al}$ in private contracts. sofisticada, mas prática, está assente a base sobre a qual se procurará construir um Direito Penal de Defesa Social ou um Direito Penal do Inimigo.

A revelação de que do outro lado dos "portugueses de bem" estão os "bandidos" soou como um alerta que nos urge a escrever em defesa do Estado de Direito Democrático, apresentando o direito penal da prevenção como resposta ao direito penal da defesa social e ao populismo penal em que este se alicerça. Para colocar em evidência a necessidade de reforçar o paradigma preventivo do direito penal, iremos começar o nosso estudo pelo papel das emoções públicas na política criminal. A partir delas conseguiremos compreender onde reside a força de uma políitica criminal que toma como seu fim a prevenção criminal, como esta está em linha com a axiologia constitucional e os modelos atuais de desenvolvimento dos Estados e, em última linha, com a compreensão da vulnerabilidade.

Através deste estudo, e das reflexões que o compõem, pretendemos oferecer um contributo para o desenvolvimento de respostas contra o populismo penal e reforçar a posição do Estado de Direito Democrático, bem como de um direito penal e política criminal construídos à imagem dos seus princípios.

This idea was first published by Gustavo Tepedino and Rodrigo da Guia Silva, who then emerged as an inspiration for the elaboration of this work, with the proposal of trying to go further. For the authors, in its original idea, Al could be used as a price fixing instrument, providing flexibility to contracts, in order to reduce the transaction costs of contracts as a result of eventual supervening facts. Al could be used as a way of controlling automatic adaptation clauses, in which the clause establishes the seller's right to revise the price originally set if the costs of 
inputs exceed a certain pre-set level. In the present work, we tried to analyze other types of contracts, such as those for lease, in which at the end of 2020 there was a significant readjustment of the IGPM, including comments on the possibility of readjusting the contracts. In this case, for example, Al could be of great help to the parties.

Palavras-chave: Inteligência Artificial; Contratos; Custos de Transação; Reajuste

Keywords: Artificial Intelligence; Contracts; Transaction Costs; Readjustment

\section{INTELIGÊNCIA ARTIFICIAL E A SUA APLICAC̣ÃO NO DIREITO}

Com o tempo, a ideia de tecnologia ganhou novos contornos e especificações e atualmente envolve uma extensa rede de pesquisadores e projetos interdisciplinares ${ }^{2}$. Com isso em mente, a noção de tecnologia é ampla e pode ser tratada de diferentes perspectivas ${ }^{3}$.

Na realidade brasileira, de forma exemplificativa, o Conselho Nacional de Justiça, através das Portarias n. 271 e 332, ambas de 2020, teve como intuito regulamentar a inteligência artificial empregada pelo Poder Judiciário ${ }^{4}$ e dispor sobre a ética, a transparência e a governança na produção e no uso de inteligência artificial no Poder Judiciário ${ }^{5}$ ○ tema da inteligência artificial ${ }^{6}$ está na ordem do dia ${ }^{7}$, um dos assuntos que mais circulam no mundo da ciência e na ambiência de uma sociedade digital em constante disrupção

\footnotetext{
2 Eduardo Magrani, A internet das coisas (Rio de Janeiro: FGV Editora, 2018), p. 30.

3 Ibid.

4 https://atos.cni.jus.br/files/original234208202012155fd949d04d990.pdf. Acesso: 30 jun. 2021

5 https://atos.cni.jus.br/files/original234208202012155fd949d04d990.pdf. Acesso: 30 jun. 2021.

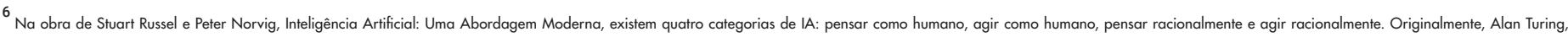

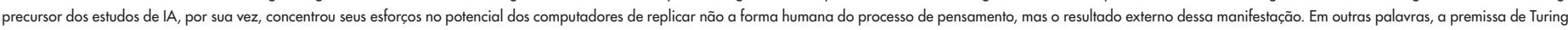
é o "jogo da imitação", no qual o computador pretende convencer o interrogador de que ele é humano e não máquina.

7 Como

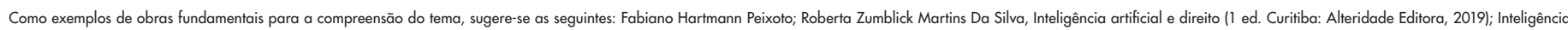

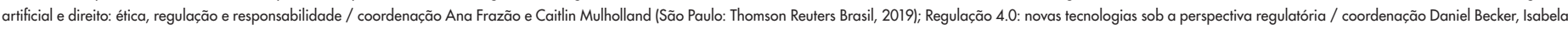

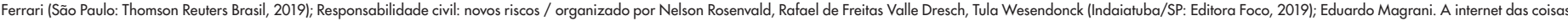

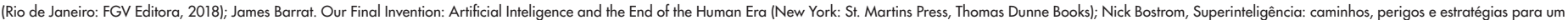
novo mundo (Rio de Janeiro: Darkside, 2018).
} 
tecnológica. Trata-se de temática de interesse e abrangência globais, independentemente da sua localização geopolítica. A interação entre o ser humano e a inteligência artificial tem sido objeto de grandes debates, especialmente no que tange a responsabilidade civil . ${ }^{8}$

Thomas Davenport, em seu artigo "From analytics to artificial intelligence", trata sobre a proximidade da analítica com o a IA:

"Machine learning is at the core of many approaches to artificial intelligence, and is analytical (i.e., statisticall) at its core. It has been employed for several decades and may be more familiar as "predictive analytics" (Siegel, 2016). Basic machine learning is predictive analytics. It uses "supervised learning" - the creation of a statistical model based on data for which the values of the outcome variable are known. For example, a machine learning model attempting to predict fraud in a bank would need to be trained on a system in which fraud has been clearly established in some cases. Machine learning can involve as simple a modeling approach as linear regression. The resulting model is tested with a validation dataset, for which the predicted outcome is compared to the known outcome. Then once a model is found that explains the variance in the training data and predicts well, it is deployed to predict or classify new data for which the outcome variable isn't known - sometimes called a scoring process". Ou seja, o que se pode verificar é que a IA não é uma temática isolada. Ela utilizou e continua utilizando das mais diversas áreas do conhecimento para ser criada e, especialmente, para se aprimorar. Por se tratar de um engenho historicamente recente /Considerado o "pai" da IA, Alan Turing publicou um famoso artigo em 1950, intitulado Computing machinery and intelligence, verdadeiro marco sobre a nova tecnologial ${ }^{10}$, acaba por suscitar alguns receios e dúvidas por parte de alguns setores da população. No Direito, essa realidade não é diferente. Existem juristas, especialmente no Brasil, que são contrários na utilização da IA ${ }^{11}$, especialmente no que diz respeito ao Poder Judiciário. Com o aprimoramento da era digital, a vida em sociedade passou a ser mais rápida e as necessidades mais urgentes. Com isso, o ânsia por soluções mais rápidas no âmbito judicial, da mesma forma, começaram a surgir. Nas palavras de Ricardo Luis Lorenzetti:

"O surgimento da era digital suscitou a necessidade de repensar importantes aspectos relativos à organização social, à democracia, à tecnologia, à privacidade, e à liberdade. $\bigcirc$ caráter aberto, interativo e global da internet, somando aos baixos custos de transação que apresenta como tecnologia, produzem um grande impacto em uma ampla categoria de questões pertencentes à sociologia jurídica e, logo, na dogmática: a noção de tempo, espaço, fronteira estatal, lugar, privacidade, bens públicos, e outras que aparecem

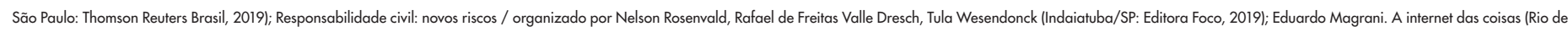

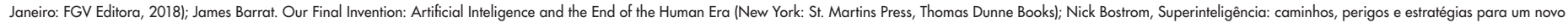
mundo (Rio de Janeiro: Darkside, 2018).

${ }^{8} \mathrm{Ch}$ 2020), pp. 301-333.

9

(D. Davenport, "From analytics to artificial intelligence", Journal of Business Analytics, 2018, p.73-80

10 Juarez Freitas, Direito e inteligência artificial: em defesa do humano (Belo Horizonte: Fórum, 2020), p. 21.

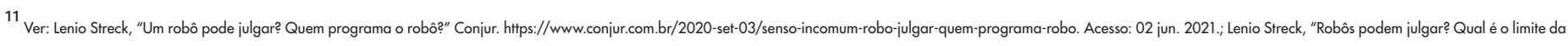
Itech-cracia?" Conjur. https://www.conjur.com.br/2020-mai-14/senso-incomum-robos-podem-julgar-qual-limite-itech-cracia. Acesso: 02 jun. 2021.

12 Ricardo Luis Lorenzetti, Teoria da decisão judicial: fundamentos de direito (São Paulo: Editora Revista dos Tribunais, 2009), p. 50.
} 
igualmente afetadas" 12. Com a inserção cada vez mais direta das novas tecnologias no Direito, alguns trâmites e formalidades antes comuns passaram a ser menos usuais, como, por exemplo, a participação em julgamentos de forma física (hoje, especialmente com a Pandemia da COVD-19, a grande maioria dos julgamentos no Brasil ocorrem de forma online). Porém, tais novidades acabam sendo objeto de críticas e, do ponto de vista acadêmico, objeto de debate. Como alerta Antonio Enrique Pérez Luño ${ }^{13}$, novas tecnologias são onipresentes na vida individual e coletiva dos seres humanos. Elas tiveram (e ainda causam) um impacto direto e decisivo no exercício da cidadania. Esse grande debate de hoje, sobre sua inserção na sociedade, traz uma profunda reflexão sobre as projeções legais e políticas decorrentes das novas tecnologias ${ }^{14}$. Em diálogo com Luño, Klaus Schwab sugere, em seu texto, que a revolução / transformação digital aumentou o poder da cognição, baseado e caracterizado pela Internet móvel, pequenos sensores eletromagnéticos e maior capacidade de inteligência artificial ${ }^{15}$. Uma dessas perspectivas é justamente o impacto direto nas relações jurídicas e, mais precisamente, no processo judicial. Lucana María Estévez Mendoza, ao dar palestras sobre o assunto, fornece o seguinte:
"Seja como for, a IA se infiltrou em nossas vidas e está pronta para permanecer nelas. Diante dessa inserção de máquinas inteligentes, parece claro que o Direito, como ciência que intervém em conflitos sociais, não pode ficar à margem, deixando a critério das partes ou grupos que defendem parcialmente seus interesses, sua resolução, embora possam fazer isso mais ou menos legitimamente." 16 Os avanços tecnológicos criam novos, presentes e futuros desafios a serem enfrentados pela sociedade e pela ciência jurídica ${ }^{17}$. A importância do tema da inteligência artificial é latente para a sociedade. As universidades, enquanto instâncias qualificadas de produção do conhecimento humano, devem ser as protagonistas neste movimento de inflexão tecnológica, e aceleradoras da inovação e da disrupção das novas tecnologias para o benefício do ser humano. $\bigcirc$ conceito de inteligência artificial, no entanto, não possui uma definição única ou pacífica entre os estudiosos sobre o assunto ${ }^{18}$. Em 19 de fevereiro de 2020, a União Europeia publicou seu "Livro Branco sobre Inteligência Artificial - Uma Abordagem Europeia para Excelência e Confiança" 19. Neste, por exemplo, o alerta que é dado é, justamente, de que "A IA é uma tecnologia estratégica que oferece muitos

\footnotetext{
13 Antonio Enrique Pérez Luño, Ciberciudadani@ o ciudadani@.com? (Gedisa, 2003), p. 57.

14 Idem.

15 Klaus Schwab, The fourth industrial Revolution (World Economic Forum, 2016), p. 11.

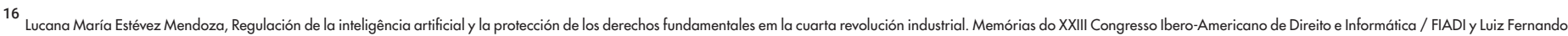
Martins Castro (organizador). (Timburi/SP: Editroa Cia do eBook, 2019), p. 267.

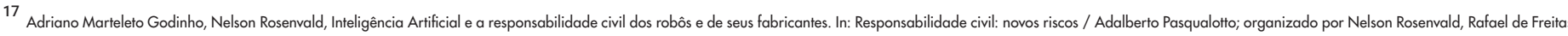
Valle Dresch, Tula Wesendonck. Indaiatuba/SP: Editora Foco, 2019, p. 23.

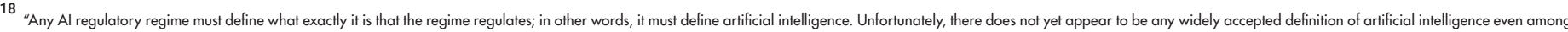

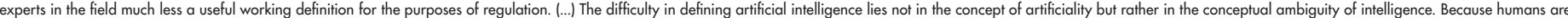

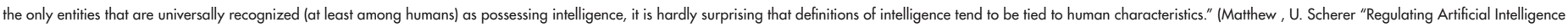
Systems: Risks, Challenges, Competencies,

19 https://op.europa.eu/en/publication-detail/-/publication/aace9398-594d-1lea-8b81-01aa75ed7lal/language-pt acessado no dia 30 de julho de 2020

20 https://op.europa.eu/en/publication-detail/-/publication/aace9398-594d-1lea-8b81-01aa75ed7lal/language-pt acessado no dia 30 de julho de 2020
} 
benefícios aos cidadãos, às empresas e à sociedade no seu conjunto, desde que seja centrada no ser humano, ética e sustentável, e respeite os direitos e os valores fundamentais" 20. Em outras palavras, a IA deve servir ao humano e não substituir o humano. Tal conclusão é de fácil compreensão para todos que analisem o "Livro Branco sobre Inteligência Artificial - Uma Abordagem Europeia para Excelência e Confiança". A ideia é que a IA não venha para substituir o humano, mas sim, para facilitar o seu trabalho e as suas tarefas diárias. De volta ao conceito, a IA foi reduzida a um conjunto de tecnologias que combinam dados, algoritmos e capacidade computacional. Nas palavras de Thomas Ramge, "para criar uma máquina inteligente, são necessários pelo menos dois elementos: uma coleção robusta de regras e um aparelho que possa processar as informações provenientes das conclusões obtidas neste banco de dados " 21. Jordi Nieva Fenoll, processualista espanhol que estuda o comportamento judicial e uma possível integração com os sistemas informatizados baseados em inteligência artificial, especialmente no que se refere à maneira de pensar do juiz e à adequação ao pensamento da máquina, alerta que: "No existe un total consenso sobre lo que significa la expressión inteligencia artificial, pero sí que podría decirse que describe la posibilidad de que las máquinas, en alguna medida, pensen, o más bien imiten el pensamiento humano a base de aprender y utilizar las generalizaciones que las personas usamos para tomar nuestras decisiones habituales" 22 . ○ autor espanhol é claro em sua afirmação. Para ele, a expressão inteligência artificial, ainda, carece de uma definição pronta e completa. Para alguns autores, tal abrangência conceitual pode, bem da verdade, ter contribuído para o seu desenvolvimento, enriquecendo os estudos sobre a própria IA. Jack Copeland, ao falar sobre o uso do termo "pensar" vinculado às máquinas, diz que nada mais é do que uma metáfora. Ele diz que é apenas uma maneira confortável de se expressar e fácil de entender . ${ }^{23}$ $\bigcirc$ autor, ainda, afirma o seguinte:

"Pero esta manera de hablar se parece, poco más o menos, a decir que mi coche decidió estropearse em el peor momento posible. Lo interessante, sin embargo, es si este estado de cosas cambiará cuando se desarrolle más la tecnologia de los computadores" ${ }^{24}$ Outra concepção (mais recente) define Al como inteligência, com a capacidade de executar tarefas específicas . ${ }^{25}$ Por exemplo, os computadores podiam jogar xadrez em 1960 e, em 1997, podiam vencer o melhor jogador de xadrez do mundo. Wolgang Hoffmann-Riem, quando discursa sobre o assunto, afirma que: "A IA é uma tecnologia transversal que visa capacitar computadores, usando grandes quantidades de

\footnotetext{
${ }^{21}$ Thomas Ramge, Who's afraid os Al? (New York/NY: The experimento, 2019), p. 32.

22 Fenol, Jordi Nieva. Inteligencia artificial y proceso judicial (Madrid: Marcial Pons, 2018), p. 20.

${ }^{23}$ Copeland, Jack. Inteligencia artificial: Una introducción filosófica (Madrid: Alianza Editorial, 1996), p. 64.

24 Idem.

${ }^{25}$ Matthew U Scherer. Regulating Artificial Intelligence, p. 360.
} 
dados (big data), capacidades computacionais apropriadas e processos específicos de análise e decisão, para alcançar realizações que se aproximam da capacidade humana ou até a excedem pelo menos em alguns aspectos" ${ }^{26}$. Atualmente, ao definir o objetivo da IA moderna, seria resolver objetivos pré-estabelecidos, componente inserido na categoria "atuação" racionalmente", definido por Stuart Russel e Peter Norvig. Esses autores, em seu trabalho, usaram o conceito de "agente racional" como uma definição de IA como aquele agente que "age como se estivesse obtendo o melhor resultado" ${ }^{27}$. Os mesmos autores também sustentam que a IA inclui tarefas como aprendizado, raciocínio, planejamento, percepção, entendimento da linguagem e robótica. É possível sintetizar os objetivos da IA como sendo a forma de alcançar aprendizado e reproduzir o modo de raciocínio da mente humana, a fim de fornecer respostas e facilitar todas as áreas de conhecimento e desempenho que o homem possa precisar, desde que presente, anteriormente, uma base de dados que possibilite a aprendizagem sobre ela ${ }^{28}$.

AlA também pode ser conceituada como a ciência e a engenharia da criação de máquinas inteligentes, especialmente programas de computador inteligentes, incluindo pesquisa e engenharia com o objetivo de usar a tecnologia digital para criar sistemas capazes de executar atividades para as quais geralmente é necessária inteligência, quando executadas por um indivíduo ${ }^{29}$. A abrangência e diferentes perspectivas da IA não param aqui. Seguimos trazendo ao leitor o que outros autores relevantes afirmam sobre a temática. Segundo Richard Bellman, a IA é a automação de atividades que associamos à cognição humana, como, por exemplo, tomada de decisão, resolução de problemas e aprendizado ${ }^{30}$. No entanto, devido ao fato de não haver unanimidade em sua conceituação, essa lacuna pode ter ajudado no crescimento do desenvolvimento do campo de estudo, pois isso não permitiu limitar a visão de seus pesquisadores ${ }^{31}$. Assim, Al seria um termo genérico. Abriga muitas áreas de estudo e técnicas, como: visão computacional, robótica, processamento de linguagem natural e aprendizado de máquina, por exemplo ${ }^{32}$. A inteligência artificial funciona a partir de sistemas de dados programados para responder de acordo com o banco de dados disponível. Esses sistemas são chamados de algoritmos. O algoritmo é um plano de ação predefinido a ser seguido pelo computador, de modo que a realização contínua de pequenas tarefas simples possibilite a execução da tarefa solicitada sem maiores gastos com trabalho humano. É uma regra usada para automatizar o processamento de dados. Eles são um conjunto finito de instruções que, por sua vez, executam uma tarefa específica ${ }^{33}$. Na raiz, um algoritmo é uma coisa pequena e simples; uma

\footnotetext{
${ }^{26}$ Wolfgang Hoffmann-Riem, "Inteligência Artificial como Oportunidade para a Regulação Jurídica", RDU, Porto Alegre, Volume 16, n. 90 (2019), p. 11-38.

27 Stuart J. Russel, Peter Norvig, Artificial Intelligence: A Modern Approach (Third Edition, Pearson Education Limited), 2016.

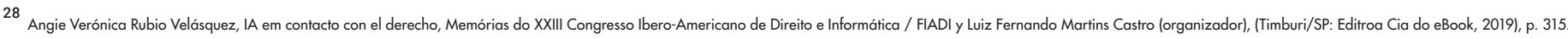

${ }^{29}$ Nilton Correia da Silva, Inteligência Artificial. In: Inteligência artificial e direito: ética, regulação e responsabilidade / coordenação Ana Frazão e Caitlin Mulholland (São Paulo: Thompson Reuters Brasil, 2019), p. 36.

30 Richard Bellman, An Introduction to Artificial Intelligence: Can Computers Think? (Boyd \& Frase, 1978).

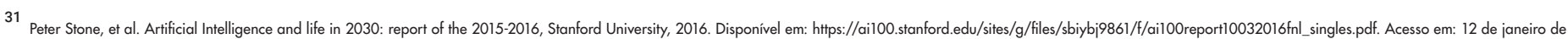
2021.

32 Fabiano Hartmann Peixoto, Inteligência artificial e direito / Fabiano Hartmann Peixoto, Roberta Zumblick Martins da Silva (1 ed. Curitiba: Alteridade Editora, 2019 ), p. 75.
} 
regra usada para automatizar o processamento de dados ${ }^{34}$. Nas palavras de Fabiano Hartmann Peixoto: "Um algoritmo pode ser definido, de maneira simplificada, como um conjunto de regras que definem com precisão uma sequência de operações, para diversos fins, como modelos de previsão, classificação, especializações" 35 . A inteligência artificial apresenta-se como o modelo mais sofisticado de algoritmo, que pode ser entendido como um conjunto de comandos visando a imitação ou simulação de decisões que devem ser adotadas por um agente humano no caso de exposição a uma situação que exige uma decisão. Nesse sentido, um conjunto de rotinas é projetado para rastrear a maneira que o sistema automatizado pode adotar para tomar uma decisão (saída). Quanto mais elaborada a maneira - a rotina programada - sem o escopo de uma árvore de decisão (ou fluxograma), mais forte é uma inteligência artificial (IA forte), enquanto quanto mais rudimentar e dependente da prática humana, mais fraco será o algoritmo. modelo do aplicativo automatizado (IA fraca) ${ }^{36}$. A IA também já tem se debruçado sobre o ramo da advocacia. Conforme destacaram John O. McGinnis e Russell G. Pearce, em artigo publicado na Fordham Law Review intitulado The great disruption: how machine intelligence will transform the role of lawyers in the delivery of legal services, serviços típicos da advocacia que são repetitivos, a exemplo de buscas por doutrina e jurisprudência, geração de documentos ou petições jurídicas e criação de cartas e memorandos serão facilmente realizados por machine learnings. Restará ao advogado as atividades em demandas complexas, em áreas altamente especializadas, bem como na atuação junto a Tribunais e em situações em que a presença humana se faz fundamental, tais como em reuniões de trabalho e de relacionamento. Destacam os autores, na conclusão do artigo: "The market for electronic legal services is at a relatively early, yet significant, stage in terms of the disruptive effect of machine intelligence in undermining lawyers' monopoly. As machine intelligence in lawyering develops exponentially, it will take an increasingly larger role in five areas of legal practice: discovery, legal search, generation of documents, creation of briefs and memoranda, and predictive analytics. Eventually, machine intelligence will prove faster and more efficient than many lawyers in providing those services. Lawyers will continue to provide services that cannot be commoditized if they are superstars, practice in highly specialized areas of law subject to rapid change, appear in court, or provide services where human relationships are central to their quality. Otherwise, no effective barriers to the advance of machine lawyering in legal practices exist-not even in the law and ethics of lawyering. Lawyers will continue to embrace machine intelligence as an input and fail to prevent nonlawyers from using it to deliver

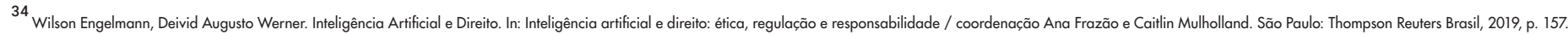

${ }^{35}$ Fabiano Hartmann Peixoto, Inteligência artificial e direito, p. 71.

${ }^{36}$ Essa construção foi formulada por meio da interpretação da distinção efetuada por Stuart J. Russel \& Peter Norvig, supra.
} 
legal services. Ultimately, therefore, the disruptive effect of machine intelligence will trigger the end of lawyers' monopoly and provide a benefit to society and clients as legal services become more transparent and affordable to consumers, and access to justice thereby becomes more widely available" ${ }^{37}$. mercado da IA no Direito ainda é incipiente. Tanto do ponto de vista extrajudicial (na sua utilização em contratos e no trabalho exercido em escritórios de advocacia), quanto na sua utilização pelo Poder Judiciário, em que a IA acaba por ter um enfoque mais voltado em auxiliar o juiz em seu julgamento. Tal possibilidade, inclusive, é objeto de diversos estudos que não será objeto do presente trabalho. Este trabalho será dedicado, mais precisamente, à matéria dos contratos, especialmente os de locação e de arrendamento, por exemplo, e de que forma a IA pode ser utilizada em seu campo.

\section{Contratos e Inteligência Artificial}

Vive-se em um mundo, em grande parte, virtual. Muitas das atividades antes exercidas única e exclusivamente pelos humanos ou foram totalmente substituídas pela máquina ou já podem ter como auxiliar algum tipo de mecanismo tecnológico que auxilia a atividade humana. Nas palavras de Jorge Castellanos Claramunt: "La tecnología impulsada por IA está introduciéndose en cada vez más aspectos de la vida de los ciudadanos, llegando las autoridades públicas a su utilización cada vez más para evaluar la personalidad o las habilidades de las personas, asignar recursos y tomar decisiones que pueden tener graves consecuencias para los derechos humanos de las personas" 38 .

Em outras palavras, a IA já está, cada vez mais,inserida no dia-a-dia de todos, até mesmo dos órgãos públicos como ferramentas de analisar tomadas de decisões que podem resultar em consequências para os direitos humanos das pessoas. Ricard Martinez se posicionar em sentido próximo: "[...] el proceso de digitalización creciente de los sectores público y privado, y las capacidades de analizar los datos mediante herramientas de machine learning gracias a las posibilidades de almacenamiento y proceso que ofrecen los entornos de cloud, favorecen la migración a un modelo de decisiones basadas en datos. La IA aporta aquí todo su valor, ya sea como herramienta de apoyo a la decisión humana asistida, ya sea como proceso automático que opera ofreciendo directamente servicios" 39 . Ou seja, o que é sustentado pelo autor é, justamente, a possibilidade da IA ser utilizada como ferramenta e apoio às decisões humanas. Novamente, voltemos ao que 0 "Livro Branco sobre Inteligência Artificial - Uma Abordagem Europeia para Excelência e Confiança" afirma. Novamente, a ideia é que a IA não venha para substituir o humano, mas sim, para facilitar o seu trabalho e as suas tarefas diárias. Para Javier Echeverría, "el objetivo principal de la tecnociência, en cambio, consiste em transformar el mundo, sea éste natural, social o artificial 40 ". E, certamente, pode-se dizer que o

\footnotetext{
37 John O. Mcginnis, Russell G Pearce, "The great disruption: how machine intelligence will transform the role of lawyers in the delivery of legal services", Fordham Law Review, [S.I.], v. 82 (2014), p. $3065-3066$.

38 Jorge Castellanos Claramunt, "Democracia, Administración Pública e Inteligencia Artificial desde uma perspectiva política y jurídica”, Revista Catalana de Dret Públic. n. 60 (2020), p. 137-147.

39 Ricard Martinez "Inteligencia artificial desde el diseño. Retos y estrategias para el cumplimiento normativo", Revista Catalana de Dret Públic. n. 58 (2019), 64-81.

40

Javier Echeverría, "Interdisciplinariedad y convergência tecnocientífica nano-bio-info-cogno", Sociologias, Porto Alegre, ano 11, n. 22, julho-dezembro (2009), p. $22-53$.
} 
mundo atual já é muito diferente do que vivíamos cerca de dez anos atrás. Hoje, em todas as esferas (seja pública ou privada) a tecnologia está presente para auxiliar ou, até mesmo, para efetivar algum serviço ou tomada de decisão. No que diz respeito às profissões atuais, a tecnologia também possui um papel fundamental. Hoje, existem profissões novas, justamente para administrar essas novas tecnologias, como profissões obsoletas, que foram praticamente extintas com $\bigcirc$ avanço tecnológico em algumas áreas. Essa é a ideia trazida por Richard Susskind: "We have immersed ourselves in books and papers about the future of professional work. And, on a daily basis, we work with leaders in various professions who are engaged in longterm thinking. There is a strong sense that the professions, as currently organized, are approaching the end of an erain the work that they do, in the identities of the providers of service, and in the nature of the service that is delivered. We are advancing into a post professional society. In the late 1990s, when dotcom fervour was rife, it was often said that one 'internet year' was like seven ordinary years, such was the speed and atmosphere of the apparent revolution. In the professions, we believe that a similar pace of change is picking up. We are struck also by how pervasive this change is likely to be-across the professions and across the globe lalthough this book has an Anglo-American focus, our exposure to other countries suggests that the trends we note will be globall" ${ }^{41}$.

mesmo autor, mais adiante em seu livro, afirma o seguinte: "The end of the professional era is characterized by four trends: the move from bespoke service; the bypassing of traditional gatekeepers; a shift from a reactive to a proactive approach to professional work; and the more-for-less challenge ". Quanto ao Direito, não importa o quão complexo ele seja, a tendência é que a inteligência artificial traga, de certa forma, automatização dos procedimentos cartorários e inovação. Automatização no sentido de que algumas atividades que antes era realizadas por servidores e estagiários passarão a serem executadas pela inteligência artificial e inovação pela mudança de paradigma na forma como essas atividades eram realizadas anteriormente. Nas palavras de Álvaro A. Sánchez Bravo: "Cada tres años se dispone de más información nueva que la creada en toda la historia de la humanidad. El único modo de gestionar esa información es mediante el uso de tecnologías digitales ${ }^{44}$ ".

mesmo autor afirma:

"El aumento de la capacidad computacional hizo que fuera possible la implementación de algoritmos cada vez más complejos, potentes y flexibles. Al mismo tiempo, la amplia disponibilidad de datos dio lugar a grandes avances en el campo de la inteligencia artificial (IA). Los datos están, por tanto, en el centro de esta transformación. Pero la forma en que se recojan y utilicen los datos debe situar los intereses de las personas en primer lugar, conforme los valores, derechos fundamentales y las normas jurídicas propias de Estados democráticos de Derecho. Uno de los más relevantes objetivos de los sistemas modernos de IA es distinguir y extraer patrones de datos sin procesar para construir su propio conocimiento. Frente a los

\footnotetext{
41 Richard Susskind, Daniel Susskind. The future of the Professions: How Technology will Transform the Work of Human Experts (1 ed. New York: Oxford University Press, 2015), p. 104-105.

42 Idem, p. 105.
} 
sistemas expertos, la solución actual no es trabajar con una base de datos de conocimiento, sino aprender conocimiento. Esa capacidad de la IA para aprender se conoce como aprendizaje de máquina y permiten que las computadoras resuelvan problema que requieren cierta comprensión del mundo real y tomen decisions situacionales y subjetivas" 45 . No Brasil, muito se tem debatido sobra e utilização da inteligência artificial no processo judicial, como forma de auxiliar 0 juiz na tomada de decisão ${ }^{46}$ e, até mesmo, na própria tomada de decisão. Porém, será que essa é a única forma de utilização da IA? Será que no âmbito privado ela não possa ser tão ou mais relevante? É nesse ponto que os autores pretendem trazer aos leitores alguns exemplos que, a princípio, podem ser objeto da implementação da IA ${ }^{47}$. Nas palavras de José Leyva Saavedra, a autonomia contratual é a possibilidade que as partes possuem de desenvolver suas próprias vontades, seus desejos, sua liberdade jurídica de contratar, no memento e na forma como desejarem, desde que de acordo com os limites do ordenamento jurídico ${ }^{48}$. Em um sistema capitalista, que reconhece como seu pontochave o princípio da liberdade da iniciativa privada, os operadores econômicos (partes do contrato) são livres para dar aos seus contratos os conteúdos concretos que considerem mais desejáveis, bem como alterá-los posteriormente para readequá-los aos diferentes e, muitas vezes, imprevisíveis cenários econômicos e sociais que estejam vivenciando. 0 regulamento contratual resulta, assim, pela vontade concorde das partes, constituindo o ponto de confluência e de equilíbrio entre os interesses - normalmente contrapostos ${ }^{49}$. A grande distinção do contrato para qualquer outro fenômeno mecanizado é a existência de alto grau de liberdade por parte dos contratantes ${ }^{50}$. Nas palavras de lan Macneil: "Contract is the projection of exchange into the future, a projection emanating from a combining in a social matrix (...)" 51 .

Foi a partir da leitura de recente artigo publicado por Gustavo Tepedino e Rodrigo da Guia Silva ${ }^{52}$ que surgiu a inspiração para debater, um pouco mais, sobre essa temática. Para os autores, a IA poderia ser utilizada como instrumento de fixação de preços, proporcionando flexibilidade aos contratos, de forma a reduzir os custos de transação dos contratos em decorrência de eventuais fatos supervenientes ${ }^{53}$. A IA poderia ser utilizada como forma de controle de cláusulas de adaptação automática, em que a cláusula estabeleça

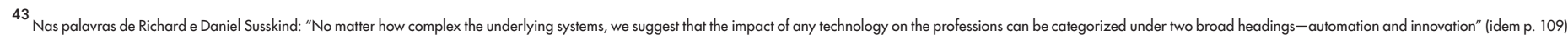

44 Álvaro A. Sánchez Bravo, "Marco Europeo para una inteligencia artificial basada en las personas" In: Derecho, Inteligencia Artificial y Nuevos Entornos Digitales. Editador por Alvaro Sánchez Bravo, 2020, p. 75.

${ }^{45}$ Idem p. 75-76.

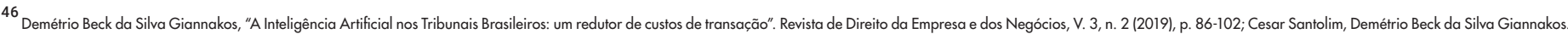
Inteligência Artificial, processo e tomada de decisão. Migalhas, https://migalhas.uol.com.br/depeso/333446/inteligencia-artificial-processo-e-tomada-de-decisao acessado em 11/01/2021.

47 .

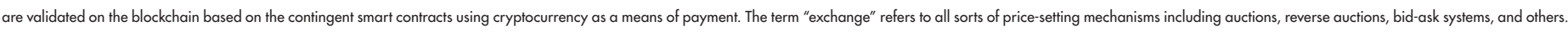

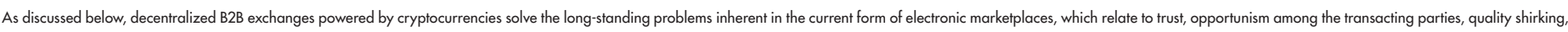

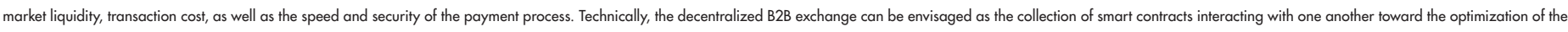

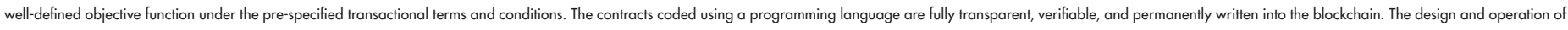

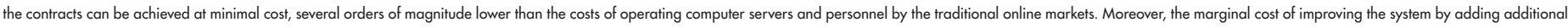

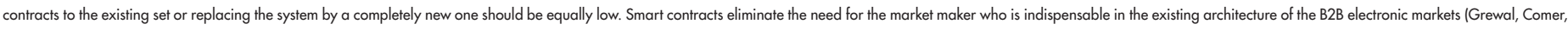
and Mehta, 2001)". (Mieszko Mazu. The Future os B2B: blockchains, smart contracts and cryptoccurencies. https://www.impgroup.org/uploads/papers/9051.pdf. Acesso: 30 jun. 2021).

48 José Leyva Saavedra, "Autonomía privada y contrato", Revista Oficial del Poder Judicial, Año 4 - 5, n. 6 y n. 7 (2010-2011), p. 282-283.
} 
o direito do vendedor à revisão do preço originariamente fixado caso os custos com insumos venham a ultrapassar certo patamar de determinada Bolsa de Valores Mobiliários ${ }^{54}$. Tal exemplo, por si só, já auxiliaria muito em casos de contratos de arrendamento, em que são, por muitas vezes, fixados com base no valor do grão da soja. Em outros casos, como os contratos de locação, pode haver uma fixação limite da correção monetária, para que o locatário não seja excessivamente prejudicado. Em caso da utilização dessa tecnologia nos contratos, acredita-se que o respeito aos termos fixados nos contratos seria ainda maior. No presente caso, haveria um sistema de IA que faria, justamente, o controle do contrato, verificando, com base no valor da soja, se o contrato poderia ser rescindido pela onerosidade excessiva ou não. No caso dos contratos de locação, poderia haver o mesmos sistema que, de forma totalmente eletrônica, controlaria o reajuste o índice IGPM podendo, em caso de alta, poderia indicar a revisão contratual. Vamos a um caso hipotético. Hoje, na cidade de Passo Fundo/RS, 60kg de soja vale R\$ 163,00. Se um fazendeiro $A$, de Passo Fundo/RS, firmasse um contrato de arrendamento com $\circ$ seu arrendatário $B$ e, em suas cláusulas, constasse uma cláusula resolutiva de que, caso o preço da soja na região ultrapasse o valor de $\mathrm{R} \$$ 180,00 a cada $60 \mathrm{Kg}$ o contrato seria rescindido automaticamente, mediante a utilização de um determinado algoritmo.
No caso dos contratos de locação, por exemplo, em outubro de 2020 já foram veiculadas informações sobre a necessidade de reajustar os contratos de locação em que foram previstos os reajustes com base no índice IGPM ${ }^{55}$. Com a alta do IGPM, os contratos de locação (residencial ou comercial) que completarão doze meses de vigência em breve terão um reajuste significativo, o que não se observava desde a época do Plano Real. Tudo isso justo no momento em que o país atravessa uma crise diante da pandemia da Covid-19. Hipoteticamente, caso nesses contratos fosse utilizado algoritmo para limitar o reajuste a determinado índice, sequer haveria a necessidade de reajuste entre as partes. A IA faria de forma automática. Sem a utilização de tal tecnologia acaba existindo espaço para a utilização por uma das partes da teoria da onerosidade excessiva. Teoria essa com origens justamente na boa-fé objetiva. Ou seja, as partes fixariam, de forma prévia, um mecanismo de resolução automática do contrato, para não depender de eventual comportamento oportunista ${ }^{56}$ de uma das partes. Tal utilização da IA poderia diminuir a discricionariedade das partes e do Estado-Juiz que, ao analisar tal contrato em ação judicial, pode determinar solução extremamente onerosa a uma das partes. Neste ponto, acredita-se que a Análise Econômica do Direito possa trazer a sua contribuição. Os litígios, ainda que bastante presentes no cotidiano daqueles que ałuam no campor direito, são vistos como um desperdício de riqueza por uma corrente de estudiosos da análise

\footnotetext{
49 Enzo Roppo, O Contrato, (Coimbra: Almedina, 2009), p. 128.

50

51 Ildem, 713. Reuters Brasil, 2020.

53 Idem, 378.

54 Idem, 383.
}

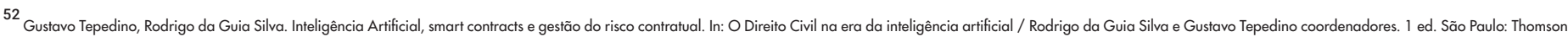


econômica. Litígios, segundo essa visão, não produzem riquezas e, portanto, significam ineficiência ${ }^{57}$. Quem litiga não está produzindo bens e serviços mas, sim, brigando pela distribuição de uma riqueza anteriormente produzida. Não sendo o bastante, o processo judicial, sob a perspectiva da AED, é dispendioso. Para além das custas e dos emolumentos recolhidos pelas partes em uma ação judicial, o Poder Judiciário é custeado e mantido por toda a sociedade, que paga impostos para, por exemplo, prover os salários dos magistrados. Simplificadamente, os custos sociais inerentes ao processo judicial são de duas ordens: os de administração e os de erro ${ }^{58}$. Sobre o segundo que pretendemos nos debruçar mais. Custos de erro são aqueles assumidos pela sociedade quando, no processo judicial, os julgadores cometem erros na aplicação do direito. Erros judiciários desvirtuam os incentivos dos agentes no mercado e na vida social como um todo, e impõem uma série de custos às partes e à sociedade. Erros judiciários criam insegurança jurídica e incentivam o ajuizamento de mais ações judiciais ${ }^{59}$. Esse é o ponto. $\bigcirc$ custo de erro é justamente o que se busca evitar. Para isso, no entender dos autores, o melhor é justamente evitar o ajuizamento de ações judiciais. Dessa forma, a aplicação da IA aos contratos pode trazer mais previsibilidade e segurança jurídica. Nessa seara, os smart contracts podem ser úteis às partes. Diante desta proposição, acredita-se estarmos tratando de, na prática, smart contracts. Para Max Raskin: "Smart contracts are defined as agreements wherein execution is automated, usually by computers. Such contracts are designed to ensure performance without recourse to the courts. Automation ensures performance, for better or worse, by excising human discretion from contract execution" 60 . Ou seja, o que se busca, bem da verdade, é justamente evitar recorrer às Cortes judiciais, afim de que a solução do caso concreto permaneça sob responsabilidade das partes contratantes. Acreditamos que evitar recorrer ao Poder Judiciário resultará na redução da discricionariedade que, no caso brasileiro, se transforma em ativismo judicial. Peguemos um caso exemplificativo. Com a ocorrência da Pandemia da COVID-19, o índice de correção IGPM sofreu aumento significativo. Diante disso, diversos locatários buscaram no Poder Judiciário a substituição do índice para ○ IPCA. Infelizmente, tal situação gerou diversos posicionamentos judiciais, em diferentes Tribunais ${ }^{61}$. Essa situação prática e concreta é, justamente, o que se busca evitar.

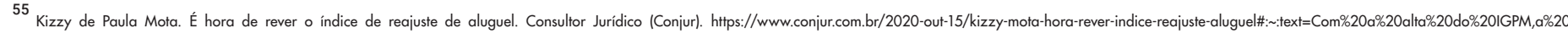
\%C3\%A9poca\%20do\%20Plano\%20Real.\&text=494\%2F64\%20veio\%20para\%20beneficiar,admitir\%20a\%20corre\%C3\%A7\%C3\%A30\%20dos\%20alugu\%C3\%A9is. Acessado em 11 jan. 2021.

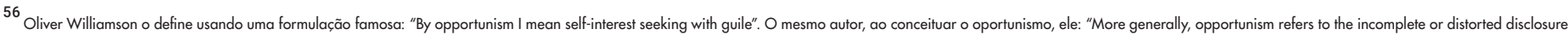

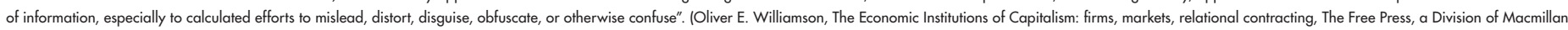
Inc, 1985, p. 47).

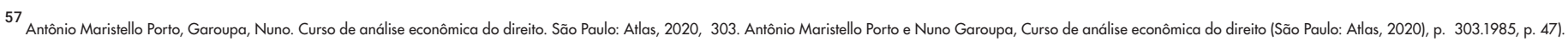

58 Idem, 316.
} 
As vantagens da utilização dessa tecnologia são muitas. Porém, talvez a mais importante seja justamente o não ajuizamento de ação judicial. Stephen Holmes e Cass R. Sunstein, em sua obra $\bigcirc$ Custo dos Direitos, identificaram muito bem uma das causas para o abarrotamento do Poder Judiciário de ações judiciais: "Uma máxima clássica da ciência jurídica diz que 'não há direito sem o remédio jurídico correspondente'" 62 . Os mesmos autores continuam:

"Os direitos têm um custo alto porque o custo dos remédios é alto. Garantir os direitos sai caro, especialmente quando essa garantia é justa e uniforme; e os direitos jurídicos não têm significado algum quando não são garantidos coercitivamente. Para dizêlo de outra maneira, quase todos os direitos implicam deveres correlativos, e os deveres só são levados a sério quando seu descumprimento é punido pelo poder público mediante recurso à fazenda pública. (...) Do ponto de vista descritivo, os direitos se reduzem a pretensões definidas e salvaguardadas pela lei" ${ }^{63}$. A utilização da inteligência artificial pode muito bem diminuir, no caso dos contratos, a busca pelo Poder Judiciário. $\bigcirc$ que se sustenta, portanto, é que a IA diminuiria a possibilidade de buscar, no Poder Judiciário, a resolução contratual, de forma a evitar que um terceiro (juiz) fique responsável de solucionar uma relação contratual dependendo da sua discricionariedade. Novamente, depositar as fichas no juiz coloca ás partes à mercê do que a AED chama de "custos de erro". Segundo Richard Susskind, existem, no momento, treze tecnologias disruptivas disponíveis para a aplicação no direito. São elas: Automação documental, conexão constante via Internet, mercados legais eletrônicos, ensino on-line, consultoria legal on-line, plataformas jurídicas abertas, comunidades online colaborativas fechadas, automatização de trabalhos repetitivos e de projetos, embedded legal knowledge, resolução on-line de conflitos, análise automatizada de documentos, previsão de resultados de processos e respostas automáticas a dúvidas legais em linguagem natural ${ }^{64}$. Os efeitos das inovações atuais e futuras só podem ser corretamente analisados e previstos a partir de uma abordagem que considere a tecnologia capaz não só de melhorar procedimentos que já existem, mas também de alterar substancialmente a forma como funciona o sistema ${ }^{65}$. Infelizmente, ainda se tem uma realidade jurisdicional muito baseada em uma ideia positivista pouco compreendida pelo Judiciário brasileiro, apostando fortemente em um papel judicial que, muitas vezes, vai além dos princípios (como, pro exemplo,

\footnotetext{
${ }^{59}$ Ibidem.

${ }^{60}$ Max Raskin, "The law and legality of smart contracts", Georgetown Law Technology Review. Vol. 1:2 (2017), p. 305-341.

${ }^{61}$ Demétrio Beck da Silva Giannakos. Uma análise sobre a substituição do IGP-M nos contratos de locação. Conjur. https://www.conjur.com.br/2021-abr-11/giannakos-substituicao-igp-contratos-locacao. Acesso: 30 jun. 2021.

${ }^{62}$ Stephen Holmes, O custo dos direitos: porque a liberdade depende de impostos / Stephen Holmes e Cass R. Sunstein; tradução de Marcelo Brandão Cipolla (São Paulo: Editora WMF Martins Fontes, 2019 ), p. 30.
} 
princípio da ampla defesa; legalidade; contraditório; boafé) existentes em nossa Constituição e na legislação vigente. Nas palavras de Antonio do Passo Cabral: "As novas teconologias têm sido utilizadas, de um lado, para estruturar bancos de dados, cadastramento e indexando as decisões judiciais tomadas (art. 979 do CPC); e, no momento aplicativo, para permitir analisar terabytes de dados para identificar o tema controverso, qual o precedente que incide no caso, e se há alguma característica que levasse à distinção (distinguishing), evitando a incidência do precedente ao caso. Em escala, o uso tecnológico de ferramentas como essas, em um sistema de precedentes, pode gerar mais segurança, coerência e igualdade, além de desestimular a litigância contrária aos precedentes e à jurisprudência consolidada. Pode ainda automaticamente levar à aplicação de outras normas que favorecem a litigância conforme os precedentes" $^{\prime \prime 6}$.

Tal situação é decorrente da incapacidade do sistema jurídico acompanhar a mutação apressada da sociedade, no qual se verifica uma veloz troca de informações através dos mais variados meios tecnológicos, economias compartilhadas e a cada momento novas formas de negócios, que tem resultado, em função de lentos passos e assoberbamento desse sistema arcaico, em uma notória insatisfação do público com o sistema jurídico e seus operadores ${ }^{67}$. Por fim, o que se sustenta no presente trabalho é justamente a possibilidade da utilização da IA em matéria contratual, com intuito de aumentar a segurança jurídica e o respeito à vontade das partes no momento da elaboração do contrato, sob pena de ser depositado, ainda mais, todas as fichas num possível ativismo judicial por parte do juiz.

\footnotetext{
${ }^{64}$ Richard Susskind, Daniel Susskind. The future of the Professions, p. 50-51.

65 Idem, pp. 43-44.

${ }^{66}$ Antonio do Passo Cabral. Processo e tecnologia: novas tendências. In: Direito, processo e tecnologia / coordenação Erik Navarro Wolkart. São Paulo: Thomson Reuters Brasil, 2020, p. 87.

${ }^{67}$ Manoel Gustavo Neubarth Trindade, Daniela Seadi Kessler, "A mediação sob o prisma da análise econômica do direito", RJLB, Ano 5, nº 4 (2019), p. 537-538.
} 


\section{Conclusões}

Não há dúvida de que a inteligência artificial chegou para ficar. Isso também se aplica à lei e aos contratos. As mudanças na sociedade e os desejos do cidadão moderno exigem de todos os envolvidos no Direito soluções mais rápidas e eficientes. Assim, torna-se importante a inserção gradual e correta da IA no Direito em geral. Cabe aos operadores do direito adaptarem-se à nova realidade. Conforme anteriormente já mencionado, é possível afirmar que a inteligência artificial se apresenta como o modelo mais avançado de algoritmo, que pode ser entendido como um conjunto de comandos visando a imitação ou simulação de decisões que devem ser adotadas por um agente humano no caso de exposição a uma situação que exija decisão. Em outras palavras, uma série de rotinas são projetadas para rastrearem a maneira que o sistema automatizado pode adotar para tomar uma determinada decisão (saída). Quanto mais elaborada a alternativa - a rotina programada - sem o escopo de uma árvore de decisão, mais robusta é uma inteligência artificial (IA forte), enquanto quanto mais rudimentar e dependente da prática humana, mais fraco será o algoritmo, modelo do aplicativo automatizado (IA fraca).
Em que sentido um contrato pode ser reajustado ou resolvido com base na inteligência artificial? Exemplos, ainda hipotéticos, de aplicação de inteligência artificial apresentados neste artigo demonstram que, para atividades em que os critérios de aplicação sejam objetivos, a máquina tende a superar a atividade humana, em eficiência ou no tempo necessário para executar tarefas.

Ou seja, em atividades que não se exija interpretação (como com a fixação de critérios de preços e de índices objetivos), nos casos hipotéticos indicados, a IA certamente traria segurança jurídica, possibilitando a criação de um critério (parâmetro) pré-definido em contrato que, ao ser alcançado, o contrato poderia ser revisado, automaticamente, pela IA lou até mesmo rescindido). A partir disso, seria possível minimizar os denominados "custos de erro" que ocorrem sempre que uma decisão é juridicamente equivocada, podendo criar incentivos indesejados e desequilibrar o mercado e a vida da sociedade como um todo. Portanto, torna-se relevante concluir que a inserção da IA em contratos privados é plenamente possível. Nesse sentido, é adequado sustentar que a aplicação da mesma pode trazer maior segurança jurídica e reduzir o comportamento oportunista das partes. 
ULPLR I ULP LAW REVIEW I REVISTA DE DIREITO DA ULP

VOL.15 I №1

DOI: $10.46294 /$ ulplr - rdulp.v15i1.7940

\section{Referências}

BARRAT, James. Our Final Invention: Artificial Inteligence and the end of the Human Era. New York: St. Martins Press, Thomas Dunne Books.

BELLMAN, Richard. An Introduction to Artificial Intelligence: Can Computers Think? Boyd \& Frase, 1978.

BOSTROM, Nick. Superinteligência: caminhos, perigos e estratégias para um novo mundo. Rio de Janeiro: Darkside, 2018.

BRAVO, Álvaro A. Sánchez. Marco Europeo para uma Inteligencia Artifical baseada em las Personas. In: Derecho, Inteligencia Artificial y Nuevos Entornos Digitales. Editador por Alvaro Sánchez Bravo, 2020

CABRAL, Antonio do Passo. Processo e tecnologia: novas tendências. In: Direito, processo e tecnologia / coordenação Erik Navarro Wolkart. São Paulo: Thomson Reuters Brasil, 2020.

CÁCERES, Enrique. Inteligencia Artificial, Derecho y E-Justice. Boletín Mexicano de Derecho Comparado. Nueva serie, año XXXIX, núm. 116, mayo-agosto de 2006, p. 593-611.

CLARAMUNT, Jorge Castellanos. Democracia, Administración Pública e Inteligencia Artificial desde uma perspectiva política y jurídica. Revista Catalana de Dret Públic. n. 60, p. 137-147, 2020.

COPELAND, Jack. Inteligencia artificial: Una introducción filosófica. Madrid: Alianza Editorial, 1996.

CORREIA DA SILVA, Nilton. Inteligência Artificial. In: Inteligência artificial e direito: ética, regulação e responsabilidade / coordenação Ana Frazão e Caitlin Mulholland. São Paulo: Thompson Reuters Brasil, 2019.

DAVENPORT, Thomas H. From analytics to artificial intelligence, Journal of Business Analytics, 2018, p.73-80.

ECHEVERRÍA, Javier. Interdisciplinariedad y convergência tecnocientífica nano-bio-info-cogno. Sociologias, Porto Alegre, ano 11 , n. 22, julho-dezembro. 2009, p. 22-53.

ENGELMANN, Wilson; WERNER, Deivid Augusto. Inteligência Artificial e Direito. In: Inteligência artificial e direito: ética, regulação e responsabilidade / coordenação Ana Frazão e Caitlin Mulholland. São Paulo: Thompson Reuters Brasil, 2019.

FENOL, Jordi Nieva. Inteligencia artificial y proceso judicial. Madrid: Marcial Pons, 2018.

FREITAS, Juarez. Direito e inteligência artificial: em defesa do humano. Belo Horizonte: Fórum, 2020.

GIANNAKOS, Demétrio Beck da Silva. A Inteligência Artificial nos Tribunais Brasileiros: um redutor de custos de transação. Revista de Direito da Empresa e dos Negócios. V. 3, n. 2 (2019), p. 86-102.

GIANNAKOS, Demétrio Beck da Silva Giannakos. Uma análise sobre a substituição do IGP-M nos contratos de locação. Conjur. https://www.conjur.com.br/2021-abr-11/giannakos-substituicao-igp-contratos-locacao. Acesso: 30 jun. 2021.

GODINHO, Adriano Marteleto; ROSENVALD, Nelson. Inteligência Artificial e a responsabilidade civil dos robôs e de seus fabricantes. In: Responsabilidade civil: novos riscos / Adalberto Pasqualotto; organizado por Nelson Rosenvald, Rafael de Freitas 
ULPLR I ULP LAW REVIEW I REVISTA DE DIREITO DA ULP

VOL.15 I №1

DOI: $10.46294 /$ ulplr - rdulp.v15i1.7940

Valle Dresch, Tula Wesendonck. Indaiatuba/SP: Editora Foco, 2019.

HARTMANN PEIXOTO, Fabiano; MARTINS DA SILVA, Roberta Zumblick. Inteligência artificial e direito. 1 ed. Curitiba: Alteridade Editora, 2019.

HOFFMANN-RIEM, Wolfgang. Inteligência Artifical como Oportunidade para a Regulação Jurídica. RDU, Porto Alegre, Volume 16, n. 90, 2019, p. 11-38, nov-dez 2019.

HOLMES, Stephen. $\bigcirc$ custo dos direitos: por que a liberdade depende de impostos / Stephen Holmes e Cass R. Sunstein; tradução de Marcelo Brandão Cipolla. São Paulo: Editora WMF Martins Fontes, 2019.

LORENZETTI, Ricardo Luis. Teoria da decisão judicial: fundamentos de direito. São Paulo: Editora Revista dos Tribunais, 2009.

MACNEIL, lan R. The many futures os contracts. Southern California Law Review. Vol. 47:691, 1973.

MAGRANI, Eduardo. A internet das coisas. Rio de Janeiro: FGV Editora, 2018.

MARTINEZ, Ricard. Inteligencia artificial desde el diseño. Retos y estrategias para el cumplimiento normativo. Revista Catalana de Dret Públic. n. 58, 64-81, 2019.

MAZU, Mieszko. The Future os B2B: blockchains, smart contracts and cryptoccurencies. https://www.impgroup.org/uploads/ papers/9051.pdf. Acesso: 30 jun. 2021.

MCGINNIS, John O.; PEARCE, Russell G. The great disruption: how machine intelligence will transform the role of lawyers in the delivery of legal services. Fordham Law Review, [S.I.], v. 82, p. 3065-3066, 2014.

MENDOZA, Lucana María Estévez. Regulación de la inteligência artificial y la protección de los derechos fundamentales em la cuarta revolución industrial. Memórias do XXIII Congresso Ibero-Americano de Direito e Informática / FIADI y Luiz Fernando Martins Castro (organizador). Timburi/SP: Editroa Cia do eBook, 2019.

MOTA, Kizzy de Paula. É hora de rever o índice de reajuste de aluguel. Consultor Jurídico (Conjur). https://www.conjur.com.br/2020out-15/kizzy-mota-hora-rever-indice-reajuste-aluguel\#: : text=Com\%20a\%20alta\%20do\%20IGPM,a\%20\%C3\%A9poca\%20 do\%2OPlano\%2OReal.\&text=494\%2F64\%20veio\%20para\%2Obeneficiar,admitir\%20a\%2Ocorre\%C3\%A7\%C3\%A30\%20dos\%20 alugu\%C3\%A9is. Acessado em 11 jan. 2021.

PÉREZ LUÑO. Antonio Enrique. Ciberciudadani@ o ciudadani@.com?. Gedisa, 2003.

PORTO, Antônio Maristello; GAROUPA, Nuno. Curso de análise econômica do direito. São Paulo: Atlas, 2020, p. 303.

RAMGE, Thomas. Who's afraid os Al? New York/NY: The experimento, 2019.

ROPPO, Enzo. O Contrato. Coimbra: Almedina, 2009.

RUSSEL, Stuart J.; NORVIG, Peter. Artificial Intelligence: A Modern Approach, Third Edition, Pearson Education Limited, 2016.

SAAVEDRA, José Leyva. Autonomía privada y contrato. Revista Oficial del Poder Judicial. Año 4 - 5, n. 6 y n. 7, $2010-2011$.

SANTOLIM, Cesar; GIANNAKOS, Demétrio Beck da Silva. Inteligência Artificial, processo e tomada de decisão. Migalhas, https://migalhas.vol.com.br/depeso/333446/inteligencia-artificial-processo-e-tomada-de-decisao acessado em 11/01/2021. 
SCHERER, Matthew U. Regulating Artificial Intelligence Systems: Risks, Challenges, Competencies, and Strategies. Harvard Journal of Law \& Technology. Volume 29, Number 2, Spring 2016.

SCHWAB, Klaus. The fourth industrial Revolution. World Economic Forum, 2016.

STONE, Peter, ET AL. Artificial Intelligence and life in 2030: report of the 2015-2016, Stanford University, 2016. Disponivel em: https://ai100.stanford.edu/sites/g/files/sbiybj9861/f/ail00report10032016fnl_singles.pdf. Acesso em: 12 de janeiro de 2021. STRECK, Lenio. Um robô pode julgar? Quem programa o robô? Conjur. https://www.conjur.com.br/2020-set-03/senso-incomumrobo-julgar-quem-programa-robo. Acesso: 02 jun. 2021.

STRECK, Lenio. Robôs podem julgar? Qual é o limite da Itech-cracia? Conjur. https://www.conjur.com.br/2020-mai-14/sensoincomum-robos-podem-julgar-qual-limite-itech-cracia. Acesso: 02 jun. 2021.

SUSSKIND, Richard; SUSSKIND, Daniel. The future of the Professions: How Technology will Transform the Work of Human Experts. 1 ed. New York: Oxford University Press, 2015.

SUSSKIND, Richard. Tomorrow's Lawyers. 2 ed. New York: Oxford University Press, 2017.

TEFFÉ, Chiara Spadaccini de; MEDON, Filipe. Responsabilidade civil e regulação de novas tecnologias: questões acerca da utilização de inteligência artificial na tomada de decisões empresariais. Revista Estudos Institucionais. V. 6, n. 1, p. 301-333, jan/ abr. 2020.

TEPEDINO, Gustavo; SILVA, Rodrigo da Guia. Inteligência Artificial, smart contracts e gestão do risco contratual. In: O Direito Civil na era da inteligência artificial / Rodrigo da Guia Silva e Gustavo Tepedino coordenadores. 1 ed. São Paulo: Thomson Reuters Brasil, 2020.

TRINDADE, Manoel Gustavo Neubarth; KESSLER, Daniela Seadi. A mediação sob o prisma da análise econômica do direito. RJLB, Ano 5, n 4, 2019.

TURING, Alan M. Computing Machinery and Intelligence. MIND a Quarterly Review of Psychology and Philosophy, Vol. LIX, n. 236, October 1950.

VELÁSQUEZ, Angie Verónica Rubio. IA em contacto con el derecho. Memórias do XXIII Congresso lbero-Americano de Direito e Informática / FIADI y Luiz Fernando Martins Castro (organizador). Timburi/SP: Editroa Cia do eBook, 2019.

WILLIAMSON, Oliver E. The Economic Institutions of Capitalism: firms, markets, relational contracting. The Free Press, a Division of Macmillan Inc, 1985. 